\title{
A physical-based atmospheric correction algorithm of unmanned aerial vehicles images and its utility analysis
}

\author{
Xiang Yu, Qing Liu, Xin Liu, Xiangyang Liu \& Yebao Wang
}

To cite this article: Xiang Yu, Qing Liu, Xin Liu, Xiangyang Liu \& Yebao Wang (2017) A physical-based atmospheric correction algorithm of unmanned aerial vehicles images and its utility analysis, International Journal of Remote Sensing, 38:8-10, 3101-3112, DOI: $\underline{10.1080 / 01431161.2016 .1230291}$

To link to this article: http://dx.doi.org/10.1080/01431161.2016.1230291

\section{曲 Published online: 28 Sep 2016.}

\section{Submit your article to this journal $๘$}

Џ Article views: 163

Q View related articles $₫$

View Crossmark data $\asymp$

4 Citing articles: 2 View citing articles 


\title{
A physical-based atmospheric correction algorithm of unmanned aerial vehicles images and its utility analysis
}

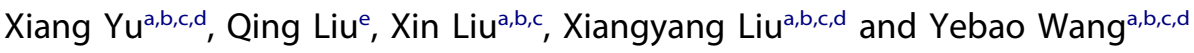

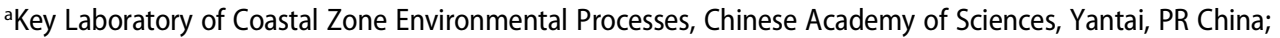 \\ bShandong Provincial Key Laboratory of Coastal Zone Environmental Processes, Yantai, PR China; 'Yantai \\ Institute of Coastal Zone Research, Chinese Academy of Sciences, Yantai, PR China; dUniversity of Chinese

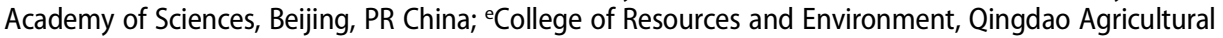 \\ University, Qingdao, PR China
}

\begin{abstract}
Unmanned aerial vehicles (UAVs)-based environmental studies are gaining space in recent years due to their advantages of minimal cost, flexibility, and very high spatial resolution. Researchers can acquire imagery according to their schedule and convenience with the option of alternating the sensors working in visible, infrared, and microwave wavelengths. The recent developments in UAVs and in the associated image-processing techniques extend the fields of UAVs application. Inherent geometric deformation of UAVs images inevitably leads to burgeoning interest in exploring the geographical registration techniques of UAVs images preprocessing. However, atmospheric correction had been generally neglected due to the low altitudes of UAVs platforms. The path radiance of low-latitude atmosphere misleads the reflectance of target objects. Thus, a valid atmospheric correction is essential in the cases where vegetation indices (VIs) are adopted in vegetation monitoring. The off-the-shelf atmospheric correction algorithms adopted in satellite-based remote sensing are typically ill-suited for UAVs-based images due to the distinctly different altitudes and radiation transfer modes. This article identified the effect of atmospheric attenuation for spectral data collected by UAVs sensors of different altitudes and developed a physical-based atmospheric correction algorithm of UAVs images. Field-measured reflectance spectrum was essential in modelling. A sunny and dry day and a flat terrain were the two prerequisites to ensure the general application of the developed algorithm. A case study was subsequently carried out to verify the utility of the developed algorithm, and the results showed that VIs based on the UAVs images of different altitudes had a similar ability in vegetation assessment as groundbased recordings. However, the assessment accuracy could be clearly improved by using the developed atmospheric correction algorithm.
\end{abstract}

\section{ARTICLE HISTORY}

Received 15 June 2016

Accepted 20 August 2016

\section{Introduction}

Remote-sensing techniques have been widely applied for all sorts of purposes, including a range of environmental monitoring and management applications. Satellite-based

CONTACT Xin Liu $\otimes$ xliu@yic.ac.cn E Yantai Institute of Coastal Zone Research, Chinese Academy of Sciences, Yantai 264003, China 
remote sensing provides regular data of large-scale, different spectral coverage and various spectral resolutions by using various sensors mounted on the associated platforms. It must be noted that the low spatial resolution of satellite remote sensing narrows down its application fields in some cases where very high spatial resolutions are required. Unmanned aerial vehicles (UAVs) provide an alternative to meet this need owing to their advantages of minimal cost, timely nature, flexibility, and very high spatial resolutions (Colomina and Molina 2014). Researchers can acquire imagery according to their schedule and convenience with the option of alternating the sensors working in visible, infrared, and microwave wavelengths and in various flight altitudes. UAVs-based environmental studies are gaining space in recent years. The recent developments in UAVs and in the associated image-processing techniques extend the UAVs application fields, such as disaster monitoring (Niethammer et al. 2012), topography (Rumpler et al. 2016; Turner, Harley, and Drummond 2016), archaeology (Alasino et al. 2012; Bendeaa et al. 2007; Remondino et al. 2011), glacier (Bhardwaj et al. 2016), soil erosion (Peter et al. 2014), precision agriculture (Comba et al. 2015; Gago et al. 2015; Pérez-Ortiz et al. 2016; Rokhmana 2015; Schellberg et al. 2008), resource management (Nishar et al. 2016), and so on.

Altitude data gained by UAVs is important in topographic surveying and mapping. Thus, the inherent geometric deformations of UAVs images inevitably lead to a burgeoning interest in exploring the geographical registration techniques of UAVs image preprocessing (Chang-Chun et al. 2011; Kekec, Yildirim, and Unel 2014; Xiang and Tian 2011; Yahyanejad and Rinner 2015). However, multispectral information dominates the application of UAVs images in vegetation monitoring, such as inter-class vegetation classification (Pérez-Ortiz et al. 2016; Tian et al. 2013), intra-class vegetation classification (Feng et al. 2015), vegetation coverage (Torres-Sánchez, López-Granados, and Peña 2015), corn lodging ( $\mathrm{Li}$ et al. 2014), and the like. Covered by geometric rectification, the atmospheric correction of multispectral UAVs images had been generally neglected.

The main reason for this status is the extremely low altitudes of UAVs platforms. Atmospheric attenuation of the very low altitude had been regarded as negligible by the mainstream of the researchers. However, atmospheric attenuation exists positively in the low-altitude atmosphere. Although small, it indeed misleads the reflectance of a target object. Thus, it should be eliminated to improve the assessment ability of UAVs-based remote sensing in the cases where accurate spectral data is necessary. Multiple spectral indices, such as the normalized excess green index (ExG) (Vol 1974), the normalized green-red difference index (NGRDI) (Gitelson et al. 2002), and the normalized difference vegetation index (NDVI), had been adopted and compared in vegetation monitoring (Rasmussen et al. 2016; Pérez-Ortiz et al. 2015a; Wang et al. 2015). However, multispectral data was straightforwardly used to calculate spectral indices in the mentioned literatures. The yielded results were questionable owing to the absence of atmospheric corrections.

The overall objectives of this study were: 1) to evaluate whether the assessment of field plot experiments using sensors mounted on UAVs was straightforward; 2) to identify the effect of atmospheric attenuation for spectral data collected by UAVs sensors of different altitudes; 3 ) to explore a general atmospheric correction technique and its application conditions; and 4) to analyse its utility by using three VIs (ExG, NGRDI, and NDVI), which are generally applied in UAVs-based remote sensing in a case study. 


\section{Materials and methods}

\subsection{Data acquisition}

The study area is located at Guangdong Zhanjiang national mangrove reserve $\left(109^{\circ}\right.$ $45^{\prime} 45^{\prime \prime}-109^{\circ} 46^{\prime} 10^{\prime \prime}, 21^{\circ} 32^{\prime} 45^{\prime \prime}-21^{\circ} 33^{\prime} 05^{\prime \prime}$ ) (Figure 1), which is flat and dominated by three species (Bruguiear gymnorrhiza, Rhizophora stylosa, and Aegiceras corniculatum). A rotary-wing (eight rotors) UAV (ZH3-600, Qingdao, China) (Figure 2) was adopted for image acquisition on 26 June 2016. For the hexacopter, vertical takeoff and landing were manually controlled using a remote control unit. The adopted Micro-Hyperspec ${ }^{\mathrm{TM}}$ VNIR (Headwall Photonics Inc., Fitchburg, USA) has a spectral range of 380-1000 nm and a spectral resolution of $5 \mathrm{~nm}$. The flights were carried out in stable ambient light conditions in the early afternoon before 14:35, with a Sun elevation angle of $45-53^{\circ}$. To identify the effect of atmospheric attenuation on spectral data collected by UAVs sensors of different altitudes, four flight heights $(10 \mathrm{~m}, 30 \mathrm{~m}, 50 \mathrm{~m}$, and $100 \mathrm{~m}$ ) were artificially set owing to the legal limits on the heights for flying UAVs (about $120 \mathrm{~m}$ ). Similar flight routes were programmed for the four flight heights $(10 \mathrm{~m}, 30 \mathrm{~m}, 50 \mathrm{~m}$, and $100 \mathrm{~m})$ and the UAV software was controlled to allow the UAV to reach the required altitudes (Figure 3). Flight speed of each flight plan was adjusted to obtain a sequence of overlapping images with $60 \%$ forward overlap and $30 \%$ side overlap. The captured geographic coordinate images were stitched by using the geo-referenced mosaic module in ENVI 4.8. Ground data was acquired with the same sensor at 31 experiment plots to obtain the spectral reflectance characteristics of the mangrove.

To identify the utility of the proposed atmospheric correction algorithm, four visible/ near-infrared (VNIR) $(480 \mathrm{~nm}, 551 \mathrm{~nm}, 675 \mathrm{~nm}$, and $851 \mathrm{~nm}$ ) were adopted to calculate ExG, NGRDI, and NDVI (Equations (1)-(3)) according to the ground spectral reflectance

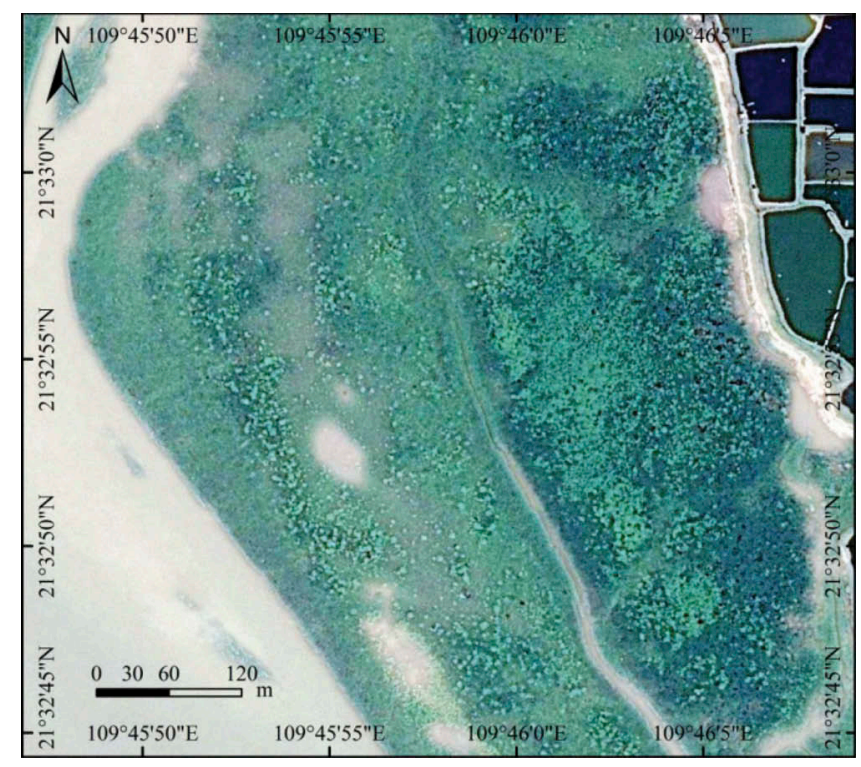

Figure 1. The experimental plot of mangrove field with a coverage of $1.38 \mathrm{k} \mathrm{m}^{2}$. 


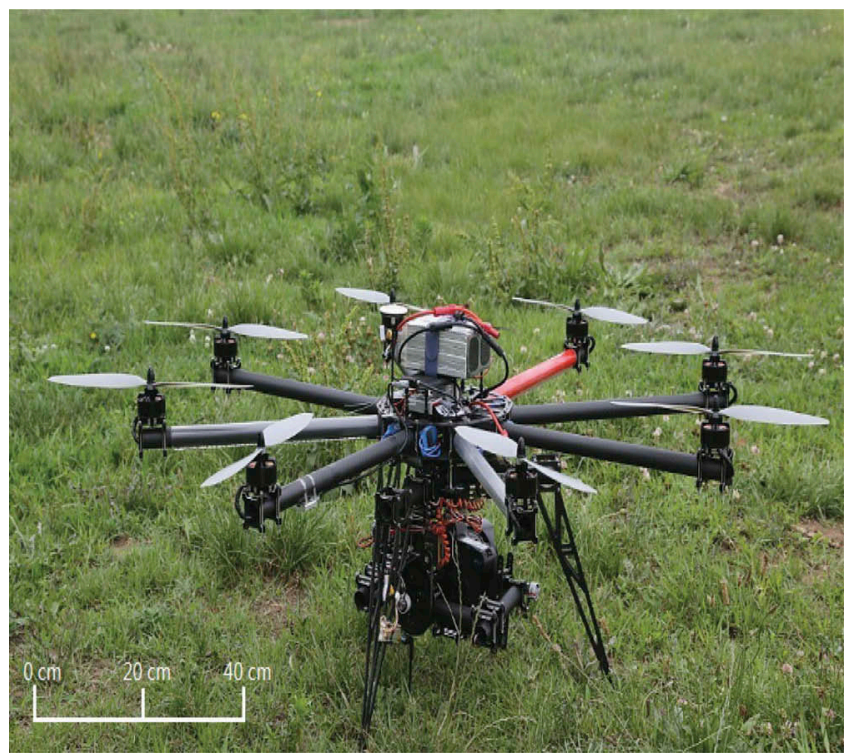

Figure 2. The rotary-wing (eight rotors) UAVs (ZH3-600, Qingdao, China).

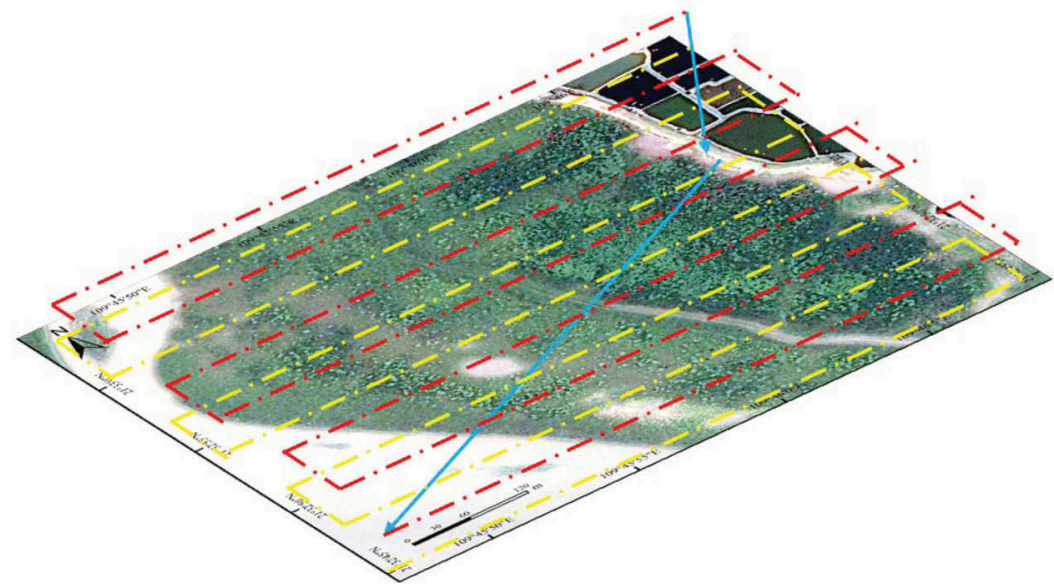

Figure 3. Sketch map of flight plans for different flight altitudes. The red dotted line represents the flight route, and the yellow dotted line represents the ground projection of flight path.

characteristics of mangroves (Figure 4). The NIR wavelength (851 nm) was generally regarded as an atmospheric window $\left(\mathrm{NIR}_{\mathrm{AW}}\right)$ with an atmospheric transmissivity $(\tau)$ of 1 (Liang 2004). Thus, it was adopted to avoid atmospheric attenuation caused by moisture absorption. Three UAV-derived VIs were compared with ground recordings and subjected to statistical analyses.

$$
\begin{gathered}
E x G=(2 G-R-B) /(R+G+B), \\
N G R D I=(G-R) /(G+R),
\end{gathered}
$$




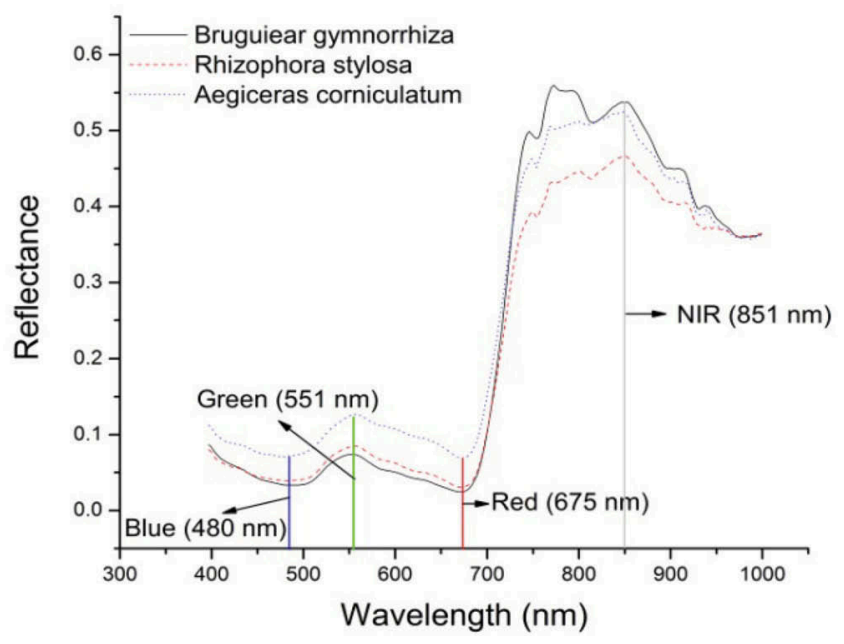

Figure 4. Spectral reflectance characteristics of mangroves.

$$
N D V I=(N I R-R) /(N I R+R)
$$

where R, G, B, and NIR denote the reflectance of red $(675 \mathrm{~nm})$, green $(551 \mathrm{~nm})$, blue $(480 \mathrm{~nm})$, and near-infrared $(851 \mathrm{~nm})$, respectively.

\subsection{Atmospheric correction algorithm}

\subsubsection{Radiation transfer mode of UAVs-based remote sensing}

UAVs platforms are characterized by their very low altitudes compared with satellite platforms. Thus, the radiation transfer modes of UAVs-based remote sensing are strikingly different from those of satellite-based remote sensing. The reflected light of the ground objects collected by UAVs sensors passes through a very thin atmosphere, which is not thicker than $120 \mathrm{~m}$. Atmospheric attenuation is composed of atmospheric absorption and atmospheric scattering. Absorptions of visible light caused by nitrogen, oxygen, and carbon dioxide are negligible. Thus, the atmospheric attenuation of visible light is mainly caused by Rayleigh scattering in the low altitude. Moreover, moisture absorptions occur at several NIR wavelengths rather than at visible wavelengths, and it is difficult to quantitatively estimate them using certain formulas. Fortunately, there are several $\mathrm{NIR}_{\mathrm{AW}}$ where moisture absorptions remain invalid and the $\tau$ values are very close to 1 (Liang 2004). Thus, when a NIR $R_{A W}$ band is involved in a UAV image and the experiment is conducted on a sunny and dry day, the proposed derivational process (Section 2.2.2) can be carried out based on the hypothesis that moisture absorption is negligible. Moreover, a sunny day makes aerosol attenuation negligible because of the lack of suspended sol particles. Thus, only atmospheric scattering was taken into account and determined, so as to be eliminated based on the given prerequisites. 


\subsubsection{Derivational process of atmospheric correction algorithm}

Digital number (DN) values of pixels in a UAV image can be calculated with the ground irradiance $(H)$, ground surface reflectance $(R), \tau$, and atmosphere path radiance $\left(D_{A}\right)$ (Equation (4)) (Li et al. 1993).

$$
\mathrm{DN}=K \times R \times \tau \times H / \pi+D_{A},
$$

where $K$ denotes the inherent proportion coefficient of the associated sensor. Then, Equation (5) is established.

$$
D_{A}=\mathrm{DN}-K \times R \times \tau \times H / \pi .
$$

$H, \tau$, and $K$ need to be derived to calculate $D_{A}$ when DN and $R$ are available. Unlike that of satellite-based remote sensing, $H$ is reasonably considered to have the same value for all of the pixels in the same UAVs image due to the uniform ground irradiance of the small ground coverage. However, this hypothesis works in a flat terrain rather than in a rugged terrain. Spatial interpolation of the measured $H$ is essential in the case of a rugged terrain (Li et al. 1993).

The difference value between the total radiation intensity and the attenuation intensity, which is defined as $\tau$, can be calculated with Lambert's law (2014) (Equation (6)).

$$
\tau_{(\lambda)}=\exp \left[-\int_{0}^{l} \beta_{(\lambda)} \times \mathrm{d} l\right]=\exp \left(-\beta_{(\lambda)} \times I\right),
$$

where $\beta_{(\lambda)}$ and I denote atmospheric attenuation coefficient at a certain wavelength and flight altitude, respectively. Atmospheric attenuation is composed of atmospheric absorption ( $k$ ) and atmospheric scattering $(\sigma)$ (Equation (7)).

$$
\beta=k_{\mathrm{m}}+\sigma_{\mathrm{m}}+k_{\mathrm{a}}+\sigma_{\mathrm{a}}
$$

where $k_{\mathrm{m}}$ and $k_{\mathrm{a}}$ denote the atmospheric absorption caused by atmospheric molecule and aerosol, respectively. Similarly, $\sigma_{\mathrm{m}}$ and $\sigma_{\mathrm{a}}$ denote atmospheric scattering caused by atmospheric molecule and aerosol, respectively. Atmospheric attenuation in the lowaltitude atmosphere is mainly caused by Rayleigh scattering due to the prerequisites discussed in Section 2.2.1. Then, Equation (8) is obtained.

$$
\beta=\sigma_{\mathrm{m}} .
$$

Moreover, there is a negative correlation between the Rayleigh scattering coefficient and the wavelength of the fourth power (Senior and Ahlgren 1973) (Equation (9)).

$$
\sigma_{\mathrm{m}}=0.00864 \times(\lambda)^{-(3.916+0.074 \times(\lambda)+(0.05 /(\lambda)))} \approx 0.00864 \times(\lambda)^{-4} .
$$

The atmospheric transmissivity ratio (Equation (10)) of different wavelengths $\left(\lambda_{i}\right.$ and $\left.\lambda_{j}\right)$ is established based on Equations (6)-(9).

$$
\begin{aligned}
\tau_{\left(\lambda_{i}\right)} / \tau_{\left(\lambda_{j}\right)} & =\exp \left(-\beta_{\left(\lambda_{i}\right)} \times I\right) / \exp \left(-\beta_{\left(\lambda_{j}\right)} \times I\right) \\
& =\exp \left(-\sigma_{\left(\lambda_{i}\right)} \times I\right) / \exp \left(-\sigma_{\left(\lambda_{j}\right)} \times I\right) .
\end{aligned}
$$


A sunny and dry day is a prerequisite in eliminating the atmospheric attenuation of NIR $R_{\text {AW }}$ caused by moisture. Thus, the path radiance of $\mathrm{NIR}_{\text {AW }}$ is negligible on a sunny day due to its extremely high transmissivity, as discussed in Section 2.2.1. Then, Equation (5) can be rewritten as follows:

$$
\mathrm{DN}_{\mathrm{NIR} \text { AW }}=K \times R_{\mathrm{NIR} \mathrm{R}_{\mathrm{AW}}} \times \tau_{\mathrm{NIR}} \times H_{\mathrm{NIR}} / \pi
$$

In addition, the $\tau$ of $\mathrm{NIR}_{\mathrm{AW}}$ is close to 1 due to the invalidation of Rayleigh scattering and atmospheric absorption. Then, Equation (12) is obtained.

$$
K \times H_{\mathrm{NIR}_{\mathrm{AW}}}=\pi \times \mathrm{DN}_{\mathrm{NIR}_{\mathrm{AW}}} /\left(R_{\mathrm{NIR}_{\mathrm{AW}}} \times \tau_{\mathrm{NIR}_{\mathrm{AW}}}\right)=\pi \times \mathrm{DN}_{\mathrm{NIR}_{\mathrm{AW}}} / R_{\mathrm{NIR}_{\mathrm{AW}}} .
$$

$H$ in the same UAVs image is defined as uniform as mentioned above. Thus, the path radiances $D_{A}$ at different wavelengths $\left(\lambda_{i}\right)$ of UAVs images can be calculated with Equation (13).

$$
\begin{aligned}
D_{A\left(\lambda_{i}\right)}= & \mathrm{DN}_{\left(\lambda_{i}\right)}-\pi \times \mathrm{DN}_{\mathrm{NIR}_{\mathrm{AW}}} / R_{\mathrm{NIR} \mathrm{RW}_{\mathrm{AW}}} \times R_{\left(\lambda_{i}\right)} \\
& \times \exp \left(-0.00864 \times\left(\lambda_{i}\right)^{-4} \times I\right) / \exp \left(-0.00864 \times \lambda_{\mathrm{NIR}_{\mathrm{AW}}}{ }^{-4} \times I\right) .
\end{aligned}
$$

\section{Results}

\subsection{Rayleigh scattering coefficients and atmospheric transmissivities of VNIR}

Rayleigh scattering coefficients vary along with wavelengths (Figure 5(a)) and atmospheric transmissivities vary along with both wavelengths and altitudes (Figure 5(b)). The effects of atmospheric attenuation on spectral data collected by UAVs sensors of different altitudes positively exist and vary along with wavelengths. Thus, atmospheric correction is essential in the application of UAVs images in the cases where accurate spectral data is demanded.
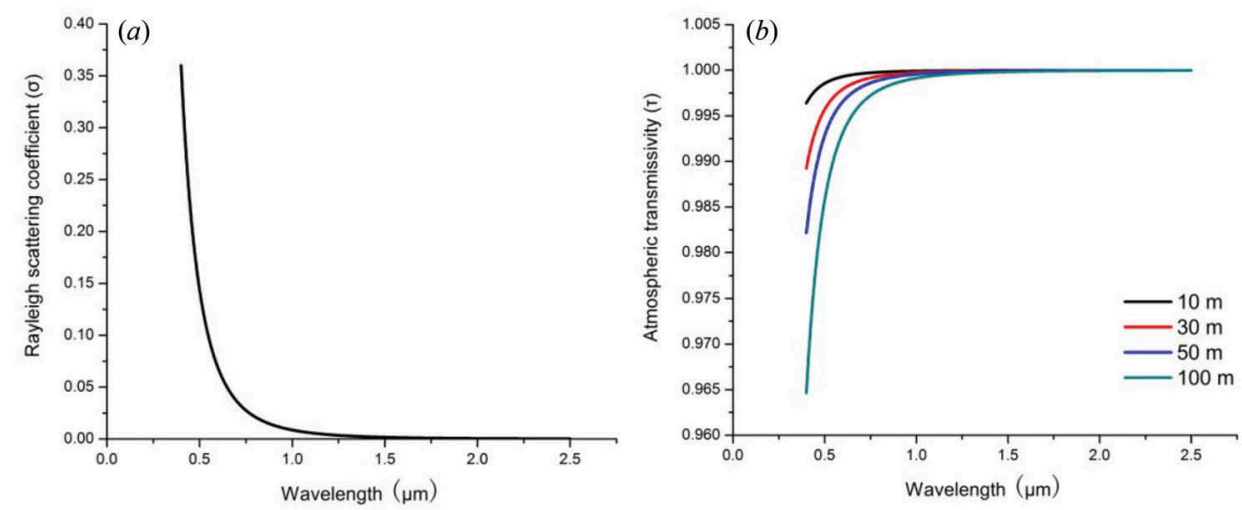

Figure 5. Rayleigh scattering coefficients (a) and atmospheric transmissivities of different altitudes (b) at visible-near-infrared wavelengths. 


\subsection{Correlation between UAVs-derived VIs and ground-derived VIs}

Three VIs of three types of mangroves at different altitudes were calculated from the original UAVs images. Similarly, the same three VIs were calculated from the atmospheric correction UAVs images (Vis_AC), which were obtained based on the yielded atmospheric correction algorithm discussed in Section 2.2.2. The differences of ExG and ExG_AC (D_ExG), NGEDI and NGEDI_AC (D_NGEDI), and NDVI and NDVI_AC (D_NDVI) at different flight altitudes $(10 \mathrm{~m}, 30 \mathrm{~m}, 50 \mathrm{~m}$, and $100 \mathrm{~m})$ are shown in Figure 6. D_ExG and D_NGEDI increased clearly along with the increase of flight altitudes. However, D_NDVI increased slightly. The results confirmed that atmospheric attenuation positively misleads the spectral data collected by UAVs sensors of different altitudes.

Correlation analysis was subsequently used to identify whether UAVs-derived VIs were straightforward. Correlation between UAVs-derived VIs and ground-derived Vlis $\left(r_{\text {Vis }}\right)$ reached a significant level $(r>0.37, p<0.05, n=31)$ (Figure $\left.7(a)\right)$. For the original UAVs images, $r_{\mathrm{ExG}}$ and $r_{\mathrm{NGRDI}}$ decreased clearly along with the increase of altitude. However, for the atmospheric correction UAVs images, $r_{\text {ExG_AC }}$ and $r_{\text {NGRDI_AC }}$ remained stable $(r=0.74$ and 0.53 ) (Figure $7(b))$. Furthermore, $r_{\text {NDVI }}$ remained stable $(r=0.70)$ irrespective of whether or not the atmospheric correction algorithm was adopted (Figure 7). The above-mentioned results show that the sensors mounted on UAVs can be directly used in the assessment of field plot experiments by using the adopted VIs; however, the assessment accuracy would be clearly improved by using the developed atmospheric correction algorithm. NDVI should be prioritized due to its robustness compared with ExG and NGRDI.

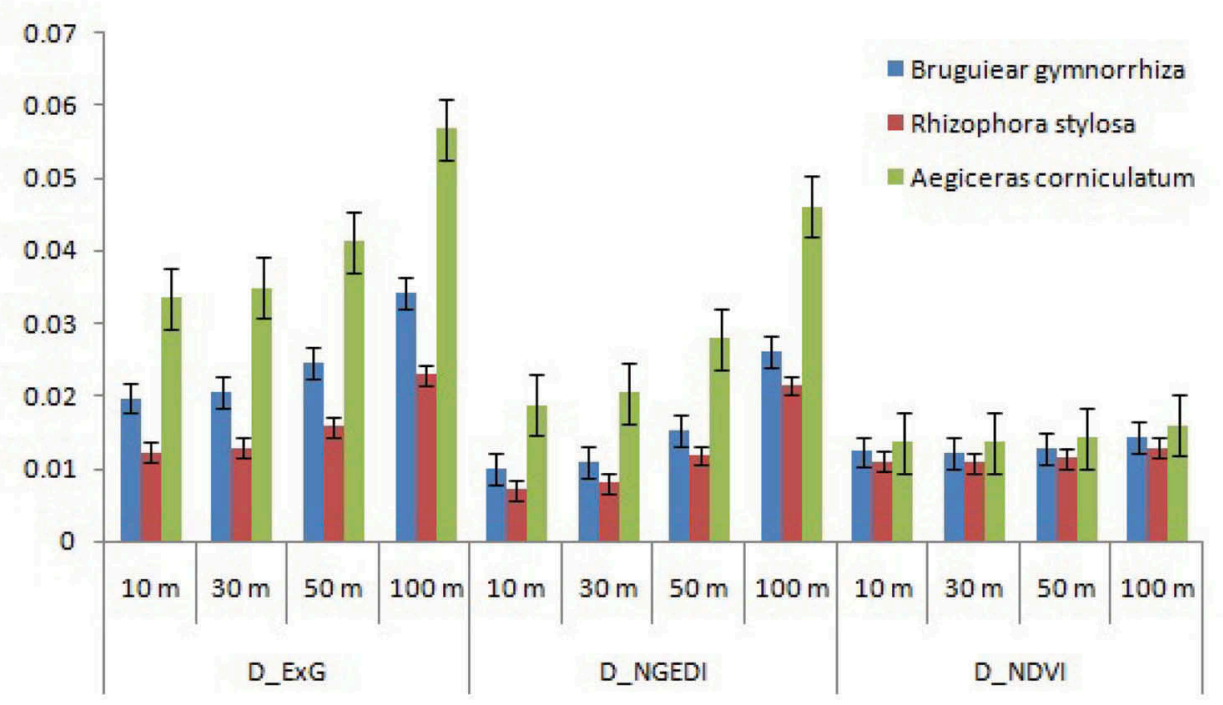

Figure 6. Differences of ExG and ExG_AC (D_ExG), NGEDI and NGEDI_AC (D_NGEDI), and NDVI and NDVI_AC (D_NDVI) at different flight altitudes $(10 \mathrm{~m}, 30 \mathrm{~m}, 50 \mathrm{~m}$, and $100 \mathrm{~m})$. 

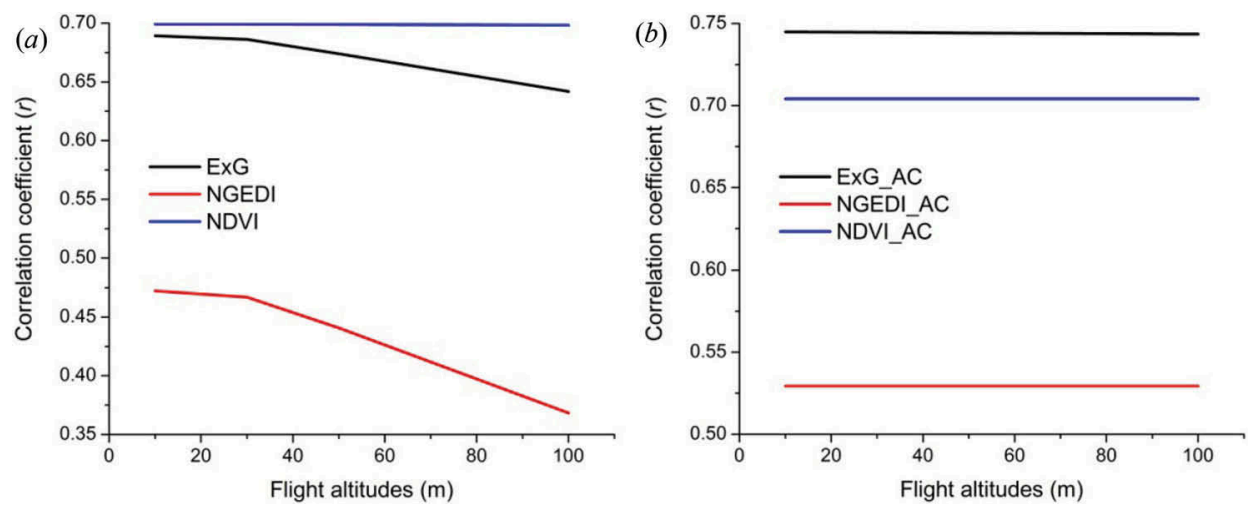

Figure 7. Correlation coefficients between the three Vls derived from the original UAVs images and the associated VIs derived from the ground recording $(a)$. Correlation coefficients between the three VIs derived from the atmospheric correction UAVs images and the associated VIs derived from the ground recording $(b)$.

\section{Discussions}

Atmospheric correction had been generally neglected due to the low altitudes of UAVs platforms in environmental monitoring. However, a case study verified that path radiance, which is mainly caused by Rayleigh scattering, positively misleads the bands reflectance of UAVs images. Rayleigh scattering coefficients vary along with wavelengths and atmospheric transmissivities vary along with both wavelengths and altitudes. The atmospheric attenuation effects on different wavelengths dominate the performances of VIs at different altitudes; thus, atmospheric correction is essential in improving the assessment accuracy of UAVs-based remote sensing.

UAVs-based remote sensing is characterized by low altitude and small ground coverage compared with satellite-based remote sensing. The uniform $H$ value of a flat terrain is essential in the developed algorithm. Thus, the algorithm does not work in a large rugged terrain. Furthermore, the proximity effect of the pixel reflectance should be avoided when selecting the modelling pixels of UAVs images. The proximity effect affects the target pixel reflectance by combining the reflectance of the surrounding adjacent pixels (Kaufman and Kaufman 1989). Thus, central pixels of a large area of the same object may be an appropriate choice to generate the accurate relationship of the $\mathrm{NIR}_{\text {AW }}$ reflectance between UAVs images pixels and field measured objects.

\section{Conclusions}

An atmospheric correction algorithm was developed to eliminate path radiance, which is mainly caused by Rayleigh scattering, for UAVs images, with NIR AW bands involved. The derivational process was carried out based on the radiation transfer mode and a reasonable set of parameters. The application conditions of the developed algorithm were raised and discussed. A sunny and dry weather condition and a flat terrain were two prerequisites to ensure the general applicability of the developed algorithm. A case study was carried out to analyse the utility of the algorithm 
by using three VIs (ExG, NGRDI, and NDVI), which are generally applied in UAVs-based remote sensing. The results showed that sensors mounted on UAVs could be directly applied in the assessment of field plot experiments; however, assessment accuracies would be clearly improved by using the developed atmospheric correction algorithm. NDVI should be given priority owing to its robustness. To the best of our knowledge, it was the first attempt to identify the effect of atmospheric attenuation for spectral data collected by UAVs sensors of different altitudes and to develop a general atmospheric correction algorithm of the UAVS images. The developed algorithm provided a reliable method to obtain accurate spectral data and improved the UAVs images' preprocessing.

\section{Disclosure statement}

No potential conflict of interest was reported by the authors.

\section{Funding}

This work is jointly supported by the funds numbered with [NSFC41371483] and [KZZD-EW-14] from the Key Research Program of the Chinese Academy of Sciences.

\section{References}

Lambert's Law. 2014. Computer Vision 443-443.

Alasino, P. H., J. A. Dahlquist, R. J. Pankhurst, C. Galindo, C. Casquet, C. W. Rapela, M. A. Larrovere, and C. M. Fanning. 2012. "Early Carboniferous Sub- to Mid-Alkaline Magmatism in the Eastern Sierras Pampeanas, NW Argentina: A Record of Crustal Growth by the Incorporation of MantleDerived Material in an Extensional Setting." Gondwana Research 22: 992-1008. doi:10.1016/j. gr.2011.12.011.

Bendeaa, H., F. Chiabrandoa, F. G. Tonolob, and D. Marenchinoa. 2007. "Mapping of Archaeological Areas Using a Low-Cost Uav the Augusta Bagiennorum Test Site." Proceedings of the XXI International CIPA Symposium, Athens, September 1-6.

Bhardwaj, A., L. Sam, A. Torres, F. J. Martín-Torres, and R. Kumar. 2016. “Uavs as Remote Sensing Platform in Glaciology: Present Applications and Future Prospects." Remote Sensing of Environment 175: 196-204. doi:10.1016/j.rse.2015.12.029.

Chang-Chun, L. I., G. S. Zhang, T. J. Lei, and A. D. Gong. 2011. "Quick Image-Processing Method of UAV without Control Points Data in Earthquake Disaster Area." Transactions of Nonferrous Metals Society of China 21: 523-528. doi:10.1016/S1003-6326(12)61635-5.

Colomina, I., and P. Molina. 2014. "Unmanned Aerial Systems for Photogrammetry and Remote Sensing: A Review." Isprs Journal of Photogrammetry \& Remote Sensing 92: 79-97. doi:10.1016/j. isprsjprs.2014.02.013.

Comba, L., P. Gay, J. Primicerio, and D. R. Aimonino. 2015. "Vineyard Detection from Unmanned Aerial Systems Images." Computers \& Electronics in Agriculture 114: 78-87. doi:10.1016/j. compag.2015.03.011.

Feng, J., K. Liu, Y. Zhu, L. I. Yong, L. Liu, and L. Meng. 2015. "Application of Unmanned Aerial Vehicles to Mangrove Resources Monitoring." Tropical Geography 35: 35-42.

Gago, J., C. Douthe, R. E. Coopman, P. P. Gallego, M. Ribas-Carbo, J. Flexas, J. Escalona, and H. Medrano. 2015. "Uavs Challenge to Assess Water Stress for Sustainable Agriculture." Agricultural Water Management 153: 9-19. doi:10.1016/j.agwat.2015.01.020. 
Gitelson, A. A., Y. J. Kaufman, R. Stark, and D. Rundquist. 2002. "Novel Algorithms for Remote Estimation of Vegetation Fraction." Remote Sensing of Environment 80: 76-87. doi:10.1016/ S0034-4257(01)00289-9.

Kaufman, Y. J., and Y. J. Kaufman. 1989. "The Atmospheric Effect on Remote Sensing and Its Correction." In Theory \& Applications of Optical Remote Sensing, edited by G. Asrar, 341. New York.

Kekec, T., A. Yildirim, and M. Unel. 2014. "A New Approach to Real-Time Mosaicing of Aerial Images." Robotics \& Autonomous Systems 62: 1755-1767. doi:10.1016/j.robot.2014.07.010.

Li, X., X. Huang, X. Wang, T. Chi, and Y. Liu. 1993. "Inverse Computation of Ground Albedo from Remotely Sensed Data." Journal of Remote Sensing 8: 306-314.

Li, Z., Z. Chen, L. Wang, J. Liu, and Q. Zhou. 2014. "Area Extraction of Maize Lodging Based on Remote Sensing by Small Unmanned Aerial Vehicle." Nongye Gongcheng Xuebao/transactions of the Chinese Society of Agricultural Engineering 30: 207-213.

Liang, S. 2004. Quantitative remote sensing of land surfaces. Wiley Praxis Series in Remote Sensing. Hoboken, NJ: Wiley \& Sons.

Niethammer, U., M. R. James, S. Rothmund, J. Travelletti, and M. Joswig. 2012. “UAV-Based Remote Sensing of the Super-Sauze Landslide: Evaluation and Results." Engineering Geology 128: 2-11. doi:10.1016/j.enggeo.2011.03.012.

Nishar, A., S. Richards, D. Breen, J. Robertson, and B. Breen. 2016. "Thermal Infrared Imaging of Geothermal Environments and by an Unmanned Aerial Vehicle (UAV): A Case Study of the Wairakei - Tauhara Geothermal Field, Taupo, New Zealand." Renewable Energy 86: 1256-1264. doi:10.1016/j.renene.2015.09.042.

Pérez-Ortiz, M., J. M. Peña, P. A. Gutiérrez, J. Torres-Sánchez, C. Hervás-Martínez, and F. LópezGranados. 2016. "Selecting Patterns and Features for Between- and Within- Crop-Row Weed Mapping Using UAV-Imagery." Expert Systems with Applications 47: 85-94. doi:10.1016/j. eswa.2015.10.043.

Pérez-Ortiz, M., J. M. Peña, P. A. Gutiérrez, J. Torres-Sánchez, C. Hervás-Martínez, and F. LópezGranados. 2015a. "A Semi-Supervised System for Weed Mapping in Sunflower Crops Using Unmanned Aerial Vehicles and A Crop Row Detection Method." Applied Soft Computing 37: 533544. doi:10.1016/j.asoc.2015.08.027.

Peter, K. D., S. d'Oleire-Oltmanns, J. B. Ries, I. Marzolff, and A. A. Hssaine. 2014. "Soil Erosion in Gully Catchments Affected by Land-Levelling Measures in the Souss Basin, Morocco, Analysed by Rainfall Simulation and UAV Remote Sensing Data." Catena 113: 24-40. doi:10.1016/j. catena.2013.09.004.

Rasmussen, J., G. Ntakos, J. Nielsen, J. Svensgaard, R. N. Poulsen, and S. Christensen. 2016. “Are Vegetation Indices Derived from Consumer-Grade Cameras Mounted on Uavs Sufficiently Reliable for Assessing Experimental Plots?" European Journal of Agronomy 74: 75-92. doi:10.1016/j.eja.2015.11.026.

Remondino, F., L. Barazzetti, F. Nex, M. Scaioni, and D. Sarazzi. 2011. "Uav Photogrammetry for Mapping and 3d Modeling - Current Status and Future Perspectives." In Proceedings of the International Conference on Unmanned Aerial Vehicle in Geomatics (UAV-g), edited by $\mathrm{H}$. Eisenbeiss, M. Kunz, and H. Ingensand, Zurich, September 14-16, 25-31.

Rokhmana, C. A. 2015. "The Potential of UAV-based Remote Sensing for Supporting Precision Agriculture in Indonesia?" Procedia Environmental Sciences 24: 245-253. doi:10.1016/j. proenv.2015.03.032.

Rumpler, M., A. Tscharf, C. Mostegel, S. Daftry, C. Hoppe, R. Prettenthaler, F. Fraundorfer, G. Mayer, and H. Bischof. 2016. "Evaluations on Multi-Scale Camera Networks for Precise and GeoAccurate Reconstructions from Aerial and Terrestrial Images with User Guidance." Computer Vision and Image Understanding. doi:10.1016/j.cviu.2016.04.008.

Schellberg, J., M. J. Hill, R. Gerhards, M. Rothmund, and M. Braun. 2008. "Precision Agriculture on Grassland: Applications, Perspectives and Constraints." European Journal of Agronomy 29: 59-71. doi:10.1016/j.eja.2008.05.005.

Senior, T., and D. Ahlgren. 1973. "Rayleigh Scattering." IEEE Transactions on Antennas \& Propagation 21: 134-134. doi:10.1109/TAP.1973.1140429. 
Tian, Z., Y. Fu, S. Liu, and A. Liufeng. 2013. "Rapid Crops Classification Based on UAV Low-Altitude Remote Sensing." Transactions of the Chinese Society of Agricultural Engineering 29 (108): 109116.

Torres-Sánchez, J., F. López-Granados, and J. M. Peña. 2015. "An Automatic Object-Based Method for Optimal Thresholding in UAV Images: Application for Vegetation Detection in Herbaceous Crops." Computers \& Electronics in Agriculture 114: 43-52. doi:10.1016/j.compag.2015.03.019.

Turner, I. L., M. D. Harley, and C. D. Drummond. 2016. "Uavs for Coastal Surveying." Coastal Engineering 114: 19-24. doi:10.1016/j.coastaleng.2016.03.011.

Vol, N. 1974. "Color Indices for Weed Identification under Various Soil, Residue, and Lighting Conditions." Proceedings of SPIE 6814: 68140Q-68140Q-68111.

Wang, X., M. Wang, S. Wang, and Y. Wu. 2015. "Extraction of Vegetation Information from Visible Unmanned Aerial Vehicle Images." Nongye Gongcheng Xuebao/transactions of the Chinese Society of Agricultural Engineering 31: 152-159.

Xiang, H., and L. Tian. 2011. "Method for Automatic Georeferencing Aerial Remote Sensing (RS) Images from an Unmanned Aerial Vehicle (UAV) Platform." Biosystems Engineering 108: 104-113. doi:10.1016/j.biosystemseng.2010.11.003.

Yahyanejad, S., and B. Rinner. 2015. "A Fast and Mobile System for Registration of Low-Altitude Visual and Thermal Aerial Images Using Multiple Small-Scale Uavs." Isprs Journal of Photogrammetry \& Remote Sensing 104: 189-202. doi:10.1016/j.isprsjprs.2014.07.015. 